\title{
Optics and radiometric magnitudes: are their connections clear?
}

\section{A. Illarramendi, A. Oleaga, J. Zubia, G. Aldabaldetreku, G. Durana, et al.}

M. A. Illarramendi, A. Oleaga, J. Zubia, G. Aldabaldetreku, G. Durana, I. Aramburu, "Optics and radiometric magnitudes: are their connections clear?," Proc. SPIE 9666, 11th Education and Training in Optics and Photonics Conference, 96661N (5 June 2009); doi: 10.1117/12.2208077

SPIE Event: Eleventh International Topical Meeting on Education and Training in Optics and Photonics, 2009, St. Asaph, United Kingdom 


\title{
Optics and radiometric magnitudes: are their connections clear?
}

\author{
M.A. Illarramendi ${ }^{1}$, A. Oleaga ${ }^{1}$, J. Zubia ${ }^{2}$, G. Aldabaldetreku², G. Durana ${ }^{2}$, and I. Aramburu ${ }^{1}$ \\ ${ }^{1}$ Departamento de Física Aplicada I, Escuela Técnica Superior de Ingeniería de Bilbao - \\ Faculty of Engineering. Universidad del País Vasco UPV/EHU. Alda Urquijo s/n E-48013 \\ Bilbao (Spain) \\ ${ }^{2}$ Departamento de Electrónica y Telecomunicaciones, Escuela Técnica Superior de \\ Ingeniería de Bilbao - Faculty of Engineering. Universidad del País Vasco UPV/EHU. Alda \\ Urquijo s/n E-48013 Bilbao (Spain)
}

\begin{abstract}
The relations between radiometric magnitudes and quantities associated to optical properties of materials (processes of reflection, transmission and emission of radiant flux by or through material media) have been analyzed. By studying some particular examples, we illustrate the dependence of optical properties of materials on the radiometric magnitude chosen and it is shown that quantities obtained from a radiometric point of view differ mathematically and physically from the corresponding Optics expressions.
\end{abstract}

\section{INTRODUCTION}

Radiometry is a system of language, mathematics and instrumentation used to describe and measure the propagation of electromagnetic (EM) radiation, including the effects on reflection, absorption, transmission and scattering by material substances. Many textbooks on electromagnetism ${ }^{1-7}$ and optical physics ${ }^{8-17}$ analyze these physical phenomena. In most of them the flux of energy associated to electromagnetic radiation is described in terms of the time average of the Poynting vector. This average is related to the square of the amplitude of the electric field and it is called "intensity" or "power density". Likewise, the reflectance and transmittance at an interface separating two different media or the reflectance and transmittance of a plane parallel plate are expressed as a function of the incident, reflected and transmitted amplitudes of the electric field. The basic concepts of radiometry are introduced in most undergraduate optics textbooks $s^{9,14,17}$. However, these texts do not explain in adequate detail the relation between the "intensity" and the radiometric magnitude called irradiance neither express the reflectance and transmittance (either at an interface or of a plate) as a function of the radiometric magnitudes. Other example where the link between both views (optical and radiometric) is far from clear is the propagation of radiation through a lossy medium. The empirical law which describes this behavior is the well-known exponential decay of the radiation with the distance. EM textbooks describe this decrease of radiation studying the decay of the amplitude of electric field with the traveled distance. However, in Optics books, there is a great dispersion in the magnitudes used to describe the exponential law and it seems that exponential decay takes always the same form regardless of the radiometric magnitude.

The purpose of this article is to contribute to a better understanding in the relation between the optical properties of materials and radiometric magnitudes, paying special attention to the physical concepts underlying the equations and trying to clarify what is somewhat messed. With that purpose in mind, in the next section radiometric magnitudes are briefly introduced. In section 3 , the definitions of reflectance and transmittance at an interface and the propagation of an elemental beam of radiation immersed in a lossy medium are analyzed as a function of radiometric magnitudes. In section 4 we develop an example which is found in most textbooks: the optical properties of plane parallel plates. The reflectance and transmittance by calculating the power fluxes at each interface of the plate have been obtained and compared with the corresponding optical expressions found in optics textbooks. Finally, we end with the conclusions. 


\section{REMARKS ON RADIOMETRIC MAGNITUDES}

Let us begin regarding the principal magnitudes used in radiometry. They are displayed in Table I. The meanings of most of the quantities are shown by their defining equations.

Table I. Radiometric magnitudes

\begin{tabular}{|c|l|l|}
\hline Symbol & Defining equation & \multicolumn{2}{c|}{ Radiometric magnitudes } \\
Name
\end{tabular}

Usually, the definition of the intensity (I) as flux per unit of solid angle, is related to point sources. However, the definition can be applied to extended surfaces using the concept of radiance (L). The intensity of an infinitesimal surface ds at $\theta$ direction respect to its normal is defined as:

$\mathrm{dl}_{\theta}=\mathrm{L} \cos \theta \mathrm{ds}$

where $L$ is the radiance at ds. The definition of $L$, stated here for a source, is extended trivially for a detector and even for a ray, at any point along its path. For a source, radiance may vary from point to point, and for a fixed point, it may vary as a function of the direction. Radiance is the most general quantity for describing the propagation of radiation through space. Its importance stems mainly from the invariance theorem that states that, in any optical system, the radiance along the path of a ray is invariant ${ }^{18}$.

Irradiance $(\mathrm{E})$ is the most important quantity for describing radiation incident on or leaving a surface when it is not essential to describe the directional distribution of that radiation in detail. It does not discriminate, for example, between very collimated radiation and radiation that is impinging from all angles. In order to take into account the orientation of the elemental surfaces in which the radiation impinges with respect to the 
direction of propagation of the beam, we propose the use of what we have called perpendicular irradiance, S, which is the radiant flux which crosses a unit area perpendicular to the direction of the flow. The definition of this perpendicular irradiance would be:

$$
\mathrm{S}=\frac{\mathrm{d} \Phi}{\mathrm{dscos} \theta}
$$

which matches the definition of the time average of the Poynting vector $S=\frac{1}{2} \sqrt{\frac{\varepsilon}{\mu}} E_{o}{ }^{2}$ where $E_{0}$ is the amplitude of the electric field.

The perpendicular irradiance is equal to the irradiance when the surface element is perpendicular to the direction of propagation of the radiation. The propagation of the radiation is frequently studied for wave planes, that is, it would correspond to a parallel beam of radiation. In this case the surface is usually placed perpendicular to the direction of propagation $(\theta=0)$, so there is no distinction between irradiance and perpendicular irradiance and both magnitudes are identical to the radiation "intensity". In this case, some Optics textbooks ${ }^{13,14,17}$ call correctly the "intensity" irradiance.

The necessity of the perpendicular irradiance will be fully revealed when, in the next sections, we proceed to develop the reflectance and transmittance coefficients as functions of radiometric magnitudes as well as in the study of the propagation of radiation in a lossy medium.

\section{PROPAGATION THROUGH AN INTERFACE SEPARATING TWO MEDIA AND A LOSSY MEDIUM}

We examine the situation where a beam of radiation passes through a smooth surface separating two media with different refractive indices $\left(n_{1}\right.$ and $\left.n_{2}\right)$. The geometric situation is shown in figure 1 . We consider the extremely thin surface region of a perfectly smooth homogeneous and isotropic dielectric material. This interface is too thin to absorb significant quantities of the radiation incident on it. The radiation incident upon the interface is split into two parts: some is reflected and the rest is transmitted. The angles of incidence and reflection $\left(\theta_{i}\right.$ and $\left.\theta_{r}\right)$ are identical due to considering a specular reflection. The conservation of energy at the interface implies:

$d^{2} \Phi_{i}=d^{2} \Phi_{r}+d^{2} \Phi_{t}$

where $d^{2} \Phi_{i}$ is the element of the incident flux on the area $d s, d^{2} \Phi_{r}$ is the element of the reflected flux and $d^{2} \Phi_{t}$ is the element of the transmitted flux. The definitions of reflectance and transmittance for incident radiation of a given spectral composition, polarization and geometrical distribution are the ratios of the reflected or transmitted flux to incident radiant flux:

$$
\rho=\frac{d^{2} \Phi_{r}}{d^{2} \Phi_{i}} \quad \text { and } \quad \tau=\frac{d^{2} \Phi_{t}}{d^{2} \Phi_{i}}
$$

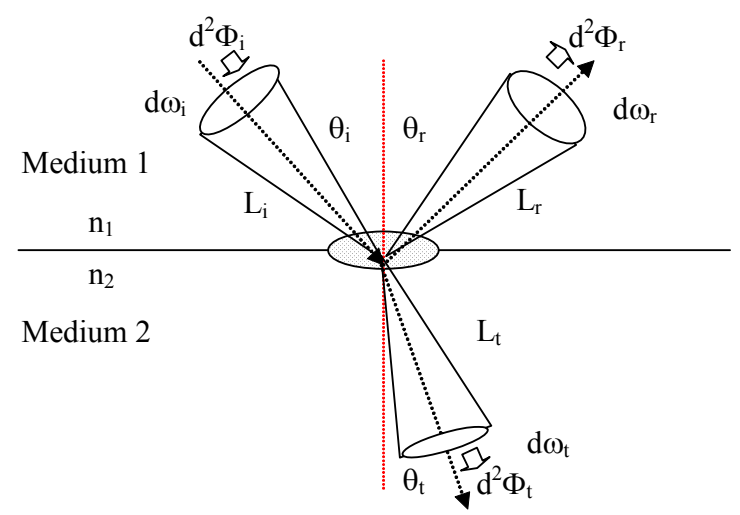

Figure 1. Reflection and transmission of an incident beam irradiating an elemental surface ds at the interface. The angles of incidence and reflection are $\theta_{\mathrm{i}}$ and $\theta_{\mathrm{r}}\left(\theta_{\mathrm{i}}=\theta_{\mathrm{r}}\right)$ and $\theta_{\mathrm{t}}$ is the angle of refraction.

Let's write them as function of the different radiometric magnitudes. By applying the definition of the corresponding radiometric magnitude and performing simple geometrical and mathematical operations, we obtain the expressions of reflectance and transmittance at an interface as a function of radiometric 
magnitudes. The obtained expressions have been depicted in Table II. Note that reflectance is always the ratio of the reflected quantity to the incident one. On the contrary, transmittance expression changes with the radiometric magnitude. Similar relations to the expressions obtained for perpendicular irradiance have been found in some texts ${ }^{14,17}$ but they simply call $S$ irradiance instead of perpendicular irradiance, what is a bit misleading. This is not the case of the text by Born ${ }^{9}$, where reflectance and transmittance are correctly defined as the ratio of irradiances $\rho=\frac{E_{r}}{E_{i}}, \tau=\frac{E_{t}}{E_{i}}$, then written in terms of $S$, and finally, in terms of the electric fields amplitudes. Although in the end, in all cases $\rho$ and $\tau$ are expressed in terms of the amplitudes, authors define them as ratios of different magnitudes. Therefore, it becomes absolutely necessary to use correctly the corresponding magnitude in order to describe accurately the optical properties of the material.

Table II. Reflectance and transmittance at an interface as a function of radiometric magnitudes. Dependence with these quantities of the exponential decay of radiation in a lossy medium.

\begin{tabular}{|c|c|c|c|c|}
\hline & ENERGY CONSERVATION & REFLECTANCE & TRANSMITTANCE & EXPONENTIAL LAW \\
\hline Flux & $d^{2} \Phi_{i}=d^{2} \Phi_{r}+d^{2} \Phi_{t}$ & $\rho=\frac{d^{2} \Phi_{r}}{d^{2} \Phi_{i}}$ & $\tau=\frac{d^{2} \Phi_{t}}{d^{2} \Phi_{i}}$ & $d^{2} \Phi_{2}=d^{2} \Phi_{1} e^{-c x}$ \\
\hline $\mathbf{L}$ & $L_{i}=L_{r}+L_{t} \frac{n_{1}^{2}}{n_{2}^{2}}$ & $\rho=\frac{L_{r}}{L_{i}}$ & $\tau=\frac{L_{t}}{L_{i}} \frac{n_{1}^{2}}{n_{2}^{2}}$ & $\mathrm{~L}_{2}=\mathrm{L}_{1} \mathrm{e}^{-\mathrm{Cx}}$ \\
\hline $\mathbf{I}$ & $\mathrm{dl}_{\theta i}=\mathrm{dl}_{\theta \mathrm{r}}+\mathrm{dl}_{\theta \mathrm{t}} \frac{\mathrm{n}_{1}^{2} \cos \theta_{\mathrm{i}}}{\mathrm{n}_{2}^{2} \cos \theta_{\mathrm{t}}}$ & $\rho=\frac{\mathrm{dl}_{\theta_{\mathrm{r}}}}{\mathrm{dl}_{\theta_{\mathrm{i}}}}$ & $\tau=\frac{\mathrm{dl}_{\theta_{\mathrm{t}}}}{\mathrm{dl}_{\theta_{\mathrm{i}}}} \frac{\mathrm{n}_{1}^{2} \cos \theta_{\mathrm{i}}}{\mathrm{n}_{2}^{2} \cos \theta_{\mathrm{t}}}$ & $\mathrm{dl}_{\theta_{2}}=\mathrm{dl}_{\theta_{1}} \frac{\left(\mathrm{ds}_{2}\right)_{\perp}}{\left(\mathrm{ds}_{1}\right)_{\perp}} \mathrm{e}^{-\mathrm{cx}}$ \\
\hline E & $\mathrm{E}_{\mathrm{i}}=\mathrm{E}_{\mathrm{r}}+\mathrm{E}_{\mathrm{t}}$ & $\rho=\frac{E_{r}}{E_{i}}$ & $\tau=\frac{E_{t}}{E_{i}}$ & $E_{2}=E_{1} \frac{\left(d s_{1}\right)}{\left(d s_{2}\right)} e^{-c x}$ \\
\hline$(\mathrm{E})_{\perp}$ & $\mathrm{S}_{\mathrm{i}}=\mathrm{S}_{\mathrm{r}}+\mathrm{S}_{\mathrm{t}} \frac{\cos \theta_{\mathrm{t}}}{\cos \theta_{\mathrm{i}}}$ & $\rho=\frac{S_{r}}{S_{i}}$ & $\tau=\frac{S_{t}}{S_{i}} \frac{\cos \theta_{t}}{\cos \theta_{i}}$ & $S_{2}=S_{1} \frac{\left(d s_{1}\right)_{\perp}}{\left(d s_{2}\right)_{\perp}} e^{-c x}$ \\
\hline
\end{tabular}

Suppose now we have some radiation leaving the surface element $\mathrm{ds}_{1}$ in the direction $\theta_{1}$ and another surface $\mathrm{ds}_{2}$ at $\mathrm{x}$ distance receiving this radiation flux from direction $\theta_{2}$. This is illustrated on Figure 2 (a). The flux entering the solid angle $d \omega_{1}$ and leaving $d s_{1}$ is $d^{2} \Phi_{1}$. The flux received by $d s_{2}$ is $d^{2} \Phi_{2}$. If the two surfaces are immersed in lossy medium whose absorption of light results from linear response, the flux falls off exponentially with increasing the distance travelled in the medium:

$$
\mathrm{d}^{2} \Phi_{2}=\mathrm{d}^{2} \Phi_{1} \mathrm{e}^{-\mathrm{cx}}
$$


here $\mathrm{c}$ is the attenuation coefficient which we suppose constant. This coefficient is the absorption coefficient when only absorbing effects are considered. If the surfaces were within a lossless medium, the flux would remain constant. By performing the appropriate calculations we can express this equation as a function of the radiometric magnitudes. The obtained equations have been included in Table II.

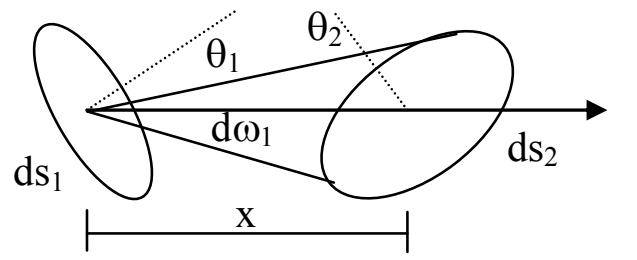

(a)

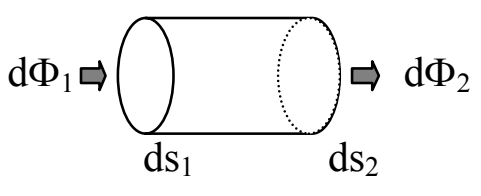

(b)

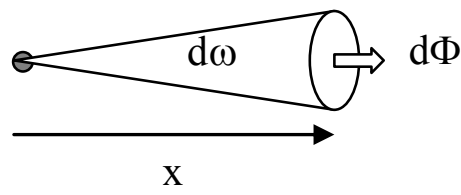

(c)

Figure 2. (a) A narrow beam of radiation that pass through the elemental areas $\mathrm{ds}_{1}$ and $\mathrm{ds}_{2}$. (b) Collimated beam propagating along the direction indicated by the arrows.(c) Point source: Radiation flux contained in the solid angle $\mathrm{d} \omega, \mathrm{d} \Phi=\operatorname{ld} \omega$.

If one has a collimated beam of radiation, that is, a bundle of approximately parallel rays propagating in the same direction with the associated flux contained in a small but measurable solid angle (Figure 2(b)), the irradiance is usually considered on a plane perpendicular to the ray. In this case, $\left(\mathrm{ds}_{1}\right)_{\perp}=d s_{1}=\left(\mathrm{ds}_{2}\right)_{\perp}=\mathrm{ds}_{2}$, the irradiance and the perpendicular irradiance, are identical and the simple exponential law is satisfied with all radiometric magnitudes. For the case of a point source immersed in a lossy medium (Figure 3(c)), it can be demonstrated that simple exponential law is satisfied if the magnitude chosen is the intensity of point source. If the magnitude used is the irradiance or the perpendicular irradiance the obtained equation is the familiar inverse-square law of radiation from a point source with the exception that the radiation is being attenuating by the medium. These results are summarized in Table III.

Table III. Exponential law of the radiation propagating within an absorbing medium applied to the case of a collimated beam of radiation and a point source. $R$ is the radius of the point source $(x>>R) \cdot I_{0}$ is the intensity of the point source and $E_{0}$ is the irradiance on the surface of the point source.

\begin{tabular}{|c|c|c|}
\hline & Collimated beam & Point source \\
\hline $\mathbf{I}$ & $\mathrm{dl}_{\theta 2}=\mathrm{dl}_{\theta 1} \exp (-\mathrm{cx})$ & $\mathrm{I}(\mathrm{x})=\mathrm{I}_{0} \exp (-\mathrm{cx})$ \\
\hline $\mathrm{E}, \mathrm{S}$ & $\mathrm{E}_{2}=\mathrm{E}_{1} \exp (-\mathrm{cx})$ & $\mathrm{E}(\mathrm{x})=\mathrm{E}_{0} \exp (-\mathrm{cx})\left(\frac{\mathrm{R}^{2}}{\mathrm{x}^{2}}\right)$ \\
\hline
\end{tabular}

To end with this section, we would like to emphasize the importance of the different cases we have studied here in order to relate properly the physical situation with the appropriate radiometric magnitude. The next section illustrates an application of the equations we have just worked out (and displayed in Table II).

\section{CASES STUDIES: PLANE PARALLEL PLATE}

A material bounded by two parallel interfaces defines an object that can reflect, transmit and absorb radiation incident on it. (Scattering processes are considered negligible). Let's now obtain the optical properties of the plate by separating the power flux at each interface into an outgoing component and an incoming component. The reflectance, transmittance and absorptance of this object are defined respectively 
as the fraction of flux incident upon the object that is reflected, transmitted and absorbed by the object for defined directions of incidence and emergence, polarization state and wavelength.

Let's consider an unpolarized collimated beam of radiation of wavelength $\lambda$ at the direction $\theta_{1}$ impinging on a plane parallel plate of a homogeneous and isotropic material of known thickness $d$ and refractive index $n$ which is surrounded by two media of index $n_{1}$ and $n_{2}$. The multiple reflections and transmissions of the incident beam are shown in Figure 3.

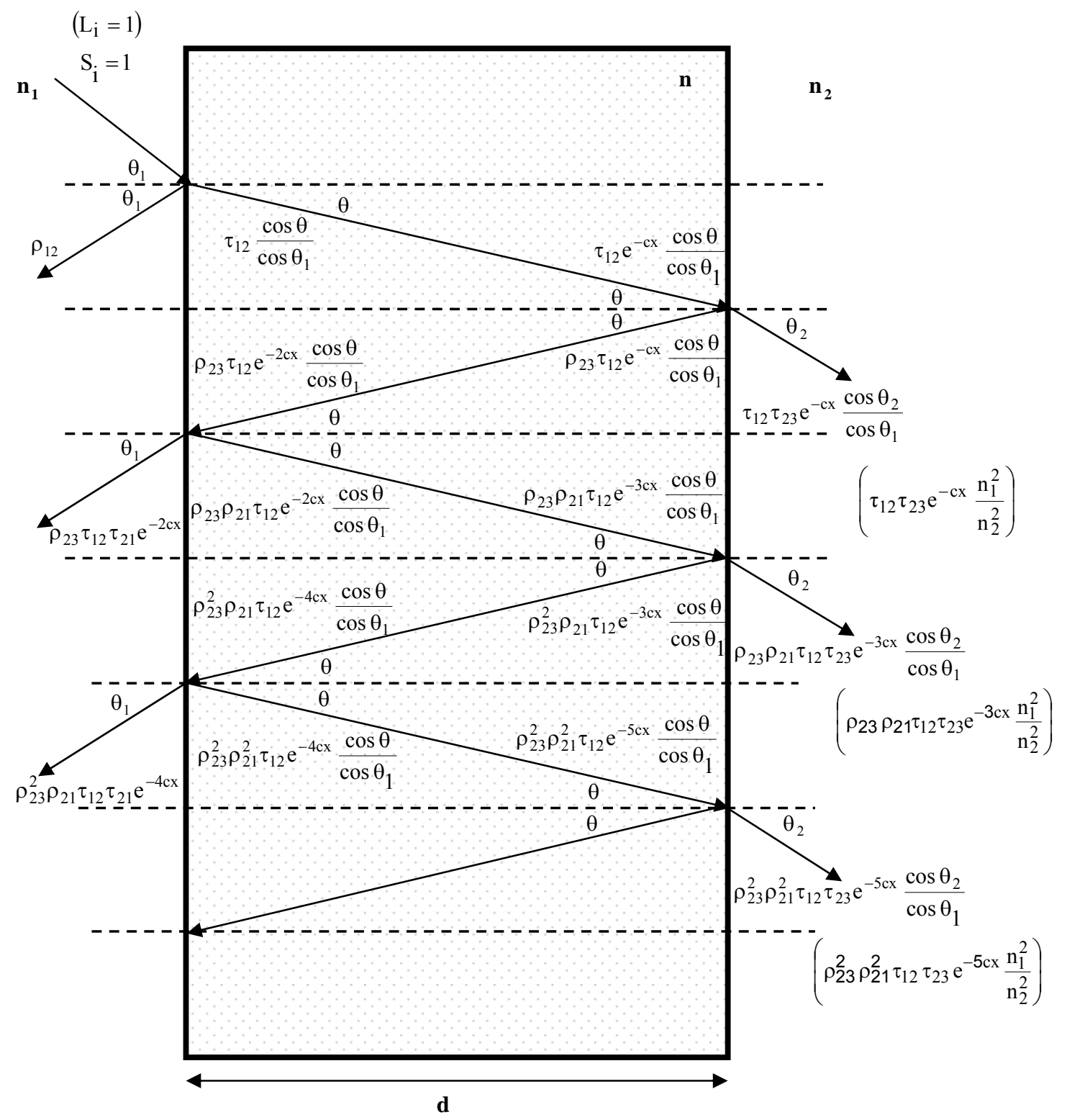

Figure 3. Multiply reflected and transmitted beams in a parallel plate. The value of $x$ is given by $x=d / \cos \theta$. The values of $S$ in different positions of the beam have been plotted for incident $S_{i}=1$. The expressions in parenthesis are the values of radiance $(L)$ for incident $L_{i}=1$.

The reflectance and transmittance of the left interface are $\rho_{12}, \tau_{12}$, and $\rho_{21}, \tau_{21}$ depending on the direction of the radiation (from $n_{1}$ medium to $n$ material or from the material to the $n_{1}$ medium). In the same way, the reflectance and transmittance of the right interface are denoted by $\rho_{23}, \tau_{23}, \rho_{32}$ and $\tau_{32}$. The definitions of 
these interface magnitudes as a function of different radiometric magnitudes have been displayed in Table II. They can be determined by using Fresnel equations, which give the ratio of reflected (or transmitted) electric field amplitude to the incident one. Absorption is considered by taking into account that the flux of the ray propagating across the material will decrease according to the exponential law.

\subsection{Radiation view}

Supposing that the incident flux is $\Phi_{1}$, the total reflectance $R$ of the plate for this situation will be the ratio of the sum of all the fluxes emerging to the left of the incident flux:

$$
\mathrm{R}=\frac{\sum_{\mathrm{k}}\left(\Phi_{\mathrm{r}}\right)_{\mathrm{k}}}{\Phi_{\mathrm{i}}}
$$

where the summatory is extended to the total number of interreflections in the material. Taking into account that the direction of reflected radiation is the same for all the emergent beams and that the angles of reflection and incidence are equal, the next relations are satisfied: $\cos \theta_{\mathrm{k}}=\cos \theta_{1} \quad \forall \mathrm{k} \Rightarrow \mathrm{d} \omega_{\mathrm{k}}=\mathrm{d} \omega_{1} \forall \mathrm{k} \Rightarrow$ $\mathrm{d} \omega_{\mathrm{k}} \mathrm{ds}_{\mathrm{k}} \cos \theta_{\mathrm{k}}=\mathrm{d} \omega_{1} \mathrm{ds} \cos \theta_{1} \forall \mathrm{k}$ (collimated beam $\mathrm{ds}_{\mathrm{k}}=\mathrm{ds} \forall \mathrm{k}$ ). Applying these relations the reflectance can be expressed as a function of different radiometric magnitudes as follows:

$$
\begin{aligned}
& R=\frac{\sum_{k}\left(\Phi_{r}\right)_{k}}{\Phi_{i}}=\frac{\sum_{k}\left(L_{r}\right)_{k} d \omega_{k} d s_{k} \cos \theta_{k}}{L_{i} d \omega_{1} d s \cos \theta_{1}}=\frac{\sum_{k}\left(L_{r}\right)_{k}}{L_{i}} \\
& R=\frac{\sum_{k}\left(\Phi_{r}\right)_{k}}{\Phi_{i}}=\frac{\sum_{k}\left(E_{r}\right)_{k} d s_{k}}{E_{i} d s}=\frac{\sum_{k}\left(E_{r}\right)_{k}}{E_{i}} \\
& \mathrm{R}=\frac{\sum_{\mathrm{k}}\left(\Phi_{\mathrm{r}}\right)_{\mathrm{k}}}{\Phi_{\mathrm{i}}}=\frac{\sum_{\mathrm{k}}\left(\mathrm{S}_{\mathrm{r}}\right)_{\mathrm{k}} \mathrm{ds} \mathrm{s}_{\mathrm{k}} \cos \theta_{\mathrm{k}}}{\mathrm{S}_{\mathrm{i}} \mathrm{ds} \cos \theta_{1}}=\frac{\sum_{\mathrm{k}}\left(\mathrm{S}_{\mathrm{r}}\right)_{\mathrm{k}}}{\mathrm{S}_{\mathrm{i}}}
\end{aligned}
$$

Therefore, the reflectance of the plate can be defined as the ratio of reflected to incident radiance or irradiance or perpendicular irradiance. Let's perform the same calculations in order to obtain the transmittance of the plate. The total transmitance $T$ of the plate will be the ratio of the sum of all the fluxes emerging to the right of the plane parallel plate:

$T=\frac{\sum_{\mathrm{k}}\left(\Phi_{\mathrm{t}}\right)_{\mathrm{k}}}{\Phi_{\mathrm{i}}}$

Now, the directions of the transmitted radiation are identical but they are different to the incident direction. The transmittance expressed as a function of different radiometric magnitudes will be:

$T=\frac{\sum_{k}\left(\Phi_{t}\right)_{k}}{\Phi_{i}}=\frac{\sum_{k}\left(L_{t}\right)_{k} d \omega_{k} d s_{k} \cos \theta_{k}}{L_{i} d \omega_{1} d s \cos \theta_{1}}$

In this case $d \omega_{k} d s_{k} \cos \theta_{k}=d \omega_{2} d s \cos \theta_{2} \forall k$ and we can write:

$$
T=\frac{d \omega_{2} \cos \theta_{2} \sum_{k}\left(L_{t}\right)_{k}}{L_{i} d \omega_{1} \cos \theta_{1}}
$$


Applying the Snell laws $n_{1} \sin \theta_{1}=n \sin \theta, n \sin \theta=n_{2} \sin \theta_{2}$ and their differential equations the expression for the transmittance is reduced to:

$T=\left(\frac{n_{1}^{2}}{n_{2}^{2}}\right) \frac{\sum_{k}\left(L_{t}\right)_{k}}{L_{i}}$

The equations for the transmittance as a function of irradiance and perpendicular irradiance will be:

$\mathrm{T}=\frac{\sum_{\mathrm{k}}\left(\Phi_{\mathrm{t}}\right)_{\mathrm{k}}}{\Phi_{\mathrm{i}}}=\frac{\sum_{\mathrm{k}}\left(\mathrm{E}_{\mathrm{t}}\right)_{\mathrm{k}} \mathrm{ds}}{\mathrm{E}_{\mathrm{i}} \mathrm{ds}}=\frac{\sum_{\mathrm{k}}\left(\mathrm{E}_{\mathrm{t}}\right)_{\mathrm{k}}}{\mathrm{E}_{\mathrm{i}}}$
$\mathrm{T}=\frac{\sum_{\mathrm{k}}\left(\Phi_{\mathrm{t}}\right)_{\mathrm{k}}}{\Phi_{\mathrm{i}}}=\frac{\sum_{\mathrm{k}}\left(\mathrm{S}_{\mathrm{t}}\right)_{\mathrm{k}} \mathrm{ds} \mathrm{s}_{\mathrm{k}} \cos \theta_{\mathrm{k}}}{\mathrm{S}_{\mathrm{i}} \mathrm{ds} \cos \theta_{1}}=\left(\frac{\cos \theta_{2}}{\cos \theta_{1}}\right) \frac{\sum_{\mathrm{k}}\left(\mathrm{S}_{\mathrm{t}}\right)_{\mathrm{k}}}{\mathrm{S}_{\mathrm{i}}}$

It can be observed that the expressions do not depend on the material refractive index (n) and the direction of the interreflections $(\theta)$ and that they are quite similar to the expressions for an interface. If the plate is surrounded by the same medium (i.e., $\mathrm{n}_{1}=\mathrm{n}_{2}$ ), the angles of incidence, reflection and transmission are equal and the reflectance (transmittance) can be defined as the ratio of reflected (transmitted) to incident radiometric magnitude, no matter which one is chosen.

Let's write the reflectance and transmittance of the plate (R and $T)$ as a function of the reflectance, transmittance of the interfaces, $\rho_{12}, \tau_{12}, \rho_{21}, \tau_{21}, \rho_{23}, \tau_{23}, \rho_{32}$ and $\tau_{32}$. For that purpose, after choosing one radiometric magnitude we must apply its corresponding equations from Table II. Figure 3 shows the values obtained when using perpendicular irradiance $(S)$ for the multiple reflections and transmissions between the two interfaces. If, for instance, radiance $L$ were chosen, the values inside the material and at the right would be different from the $S$ values displayed on figure 3 , while the $L$ values at the left of the figure would not change.

The exponential law for the decreasing of radiation has been applied in the propagation of the beam inside the material. In this case this law does not change with the radiometric magnitude and takes its simplified expression (for instance, $S_{2}=S_{1} \exp (-c x)$ due to $\left.\left(d_{1}\right)_{\perp}=\left(d s_{2}\right)_{\perp}\right)$. By performing mathematical operations the following expressions for $\mathrm{R}$ and $\mathrm{T}$ are obtained:

$$
R=\rho_{12}+\frac{\rho_{23} \tau_{12} \tau_{21} e^{-2 c x}}{1-\rho_{21} \rho_{23} e^{-2 c x}} \text { and } T=\frac{\tau_{12} \tau_{23} e^{-c x}}{1-\rho_{12} \rho_{23} e^{-2 c x}} \frac{\cos \theta_{2}}{\cos \theta_{1}}
$$

and the law of energy conservation implies that the absorptance $A$ of the plate is given by $A=1-R-T$.

In the case that the external medium be the same $\left(n_{1}=n_{2}\right.$ and $\left.\cos \theta_{1=} \cos \theta_{2}\right)$, using Fresnel equations we get $\rho_{23}=\rho_{21}=\rho_{12}=\rho, \tau_{12} \tau_{21}=(1-\rho)^{2}$, so that equations $(16)$ are simplified to:

$R=\rho+\frac{\rho(1-\rho)^{2} e^{-2 c x}}{1-\rho^{2} e^{-2 c x}}$ and $\quad T=\frac{(1-\rho)^{2} e^{-2 c x}}{1-\rho^{2} e^{-2 c x}}$

If absorption processes are neglected $\left(e^{-c x} \approx 1\right)$, the result is: and

$$
\mathrm{T}_{\mathrm{rad}}=\frac{1-\rho}{1+\rho}
$$


These magnitudes, that we call radiation reflectance and transmittance, only depend on $\rho$, that is, on the incident angle and the refraction indexes.

\subsection{Optical view}

Let's pay attention to the corresponding expressions that appear in many Optics textbooks for the last case (i.e., same external medium $n_{1}=n_{2}$ ); these books ${ }^{9,10,17}$ usually provide the total reflected and transmitted "intensity", from which the reflectance and transmittance of the plate are easily calculated by performing the corresponding ratios. The reflection and transmission expressions of a plate when absorption processes are negligible are given by:

$$
\begin{aligned}
& R=\frac{(2-2 \cos \delta) r_{12}^{2}}{1+r_{12}^{4}-2 r_{12}^{2} \cos \delta} \text { with } \quad\left|r_{12}\right|=\left|r_{21}\right| \\
& T=\frac{\left(t_{12} t_{21}\right)^{2}}{1+r_{12}^{4}-2 r_{12}^{2} \cos \delta} \text { with } t_{12} t_{21}=1-r_{12}^{2}
\end{aligned}
$$

where $\delta$ is the phase difference of two consecutive waves $\left(\delta=\frac{4 \pi}{\lambda} n d \cos \theta\right)$, and $r$ and $t$ are reflection and transmission coefficients; the latter are defined as the ratio of reflected/transmitted electric field amplitudes to the incident electric field amplitude, $r=\frac{\left(E_{0}\right)_{r}}{\left(E_{0}\right)_{i}}, t=\frac{\left(E_{0}\right)_{t}}{\left(E_{0}\right)_{i}}$, and they are determined by applying the Fresnel equations. Taking into account the definition of $S$ as a function of the electric field amplitude, considering nonmagnetic materials and using properly the definitions displayed in Table II, the correct values for $\rho, \tau_{12}, \tau_{21}$ are obtained in this case as $\rho=r_{12}^{2}=r_{21}^{2}, \tau_{12}=\frac{n \cos \theta}{n_{1} \cos \theta_{1}} t_{12}^{2}, \quad \tau_{21}=\frac{n_{1} \cos \theta_{1}}{n \cos \theta} t_{21}^{2}$. We can then transform equations (19)-(20) into:

$$
\mathrm{R}_{\mathrm{opt}}=\frac{2(1-\cos \delta) \rho}{1+\rho^{2}-2 \rho \cos \delta} \quad \text { and } \quad \mathrm{T}_{\mathrm{opt}}=\frac{(1-\rho)^{2}}{1+\rho^{2}-2 \rho \cos \delta}
$$

\subsection{Comparison}

Let's compare now equations (18) and (21). Obviously, they are not the same. It can be noticed in equations (21) the dependence on the phase difference of two consecutive waves which implies that these expressions depend on the thickness of the plate. On the contrary, equations (18) do not depend on the thickness. As an example, we plot in Figure 4 both transmittances as a function of thickness for an uncoated calcium fluoride window at $486 \mathrm{~nm}^{19}$ at two angles of incidence. Calcium fluoride has very low absorption at this wavelength, so the equations (18) and (21) are appropriate for this case. The curved lines are values obtained from eq. (21) and the straight line from eq. (18). As it can be seen, the optical values oscillate around those calculated from the radiation method and the oscillations change with the value of angle of incidence. The maximum dispersion of the $R_{\text {opt }}$ and $T_{\text {opt }}$ values is $\Delta R_{o p t}=\Delta T_{o p t}=4 \rho /(1+\rho)^{2}$, which only depends on $\rho$. In Figure 5, it can be seen the dependence of both transmittances ( $T_{\text {rad }}$ and $T_{\text {opt }}$ ) and the dispersion $\Delta T_{\text {opt }}$ with the angle of incidence. The dispersion is constant and small at low angles and increases strongly at higher angles of incidence. As we have noticed in the previous figure, the $T_{\text {opt }}$ values oscillate around the $T_{\text {rad }}$ values. How can we explain from a physical point of view these results? 


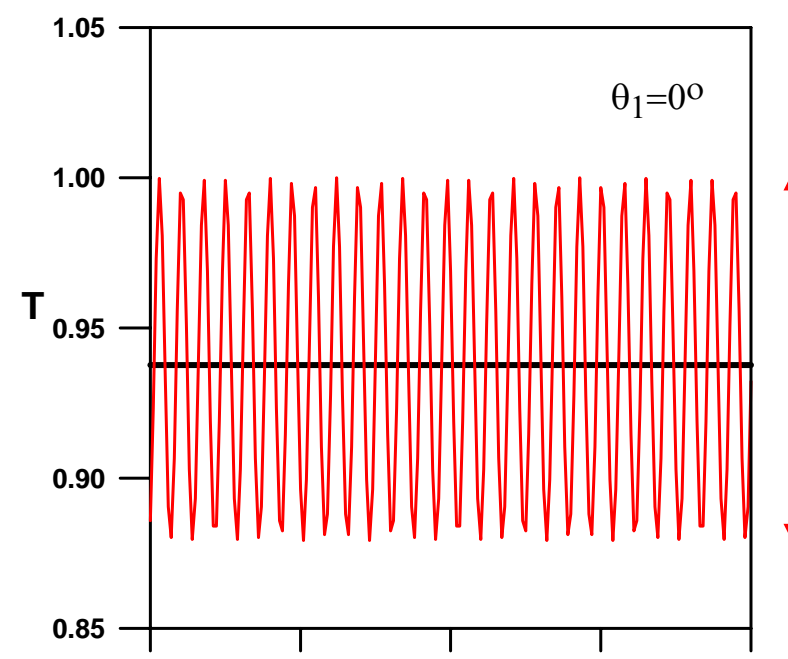

$\Delta \mathbf{T}_{\text {opt }}$

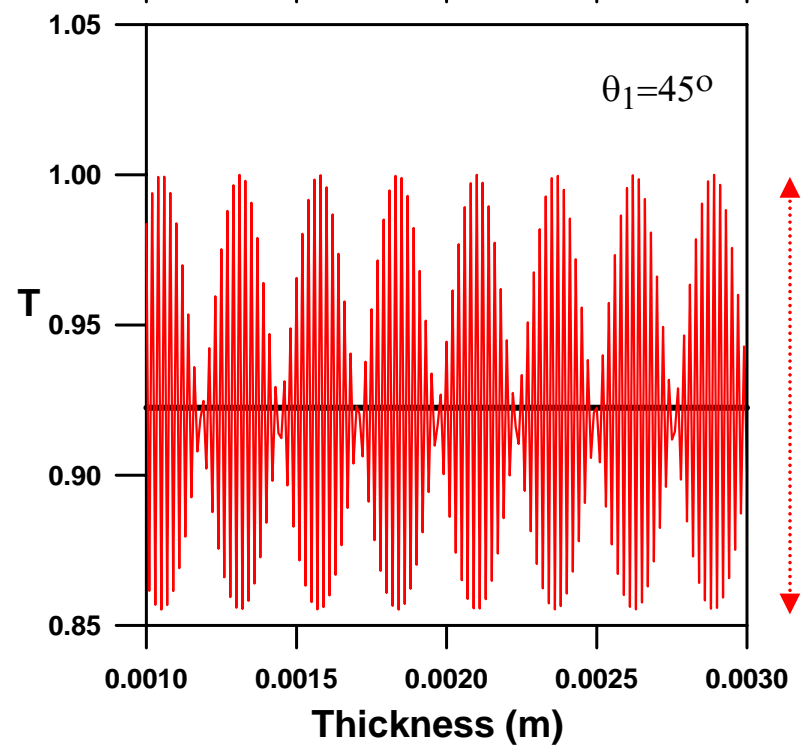

Figure 4. Transmittance values for an uncoated calcium fluoride window at $486 \mathrm{~nm}$ as a function of thickness at normal incidence (a) and at incidence $\theta_{1}=45^{\circ}$ (b). The curved lines are the optical values and the black straight lines are $T_{r a d}$.

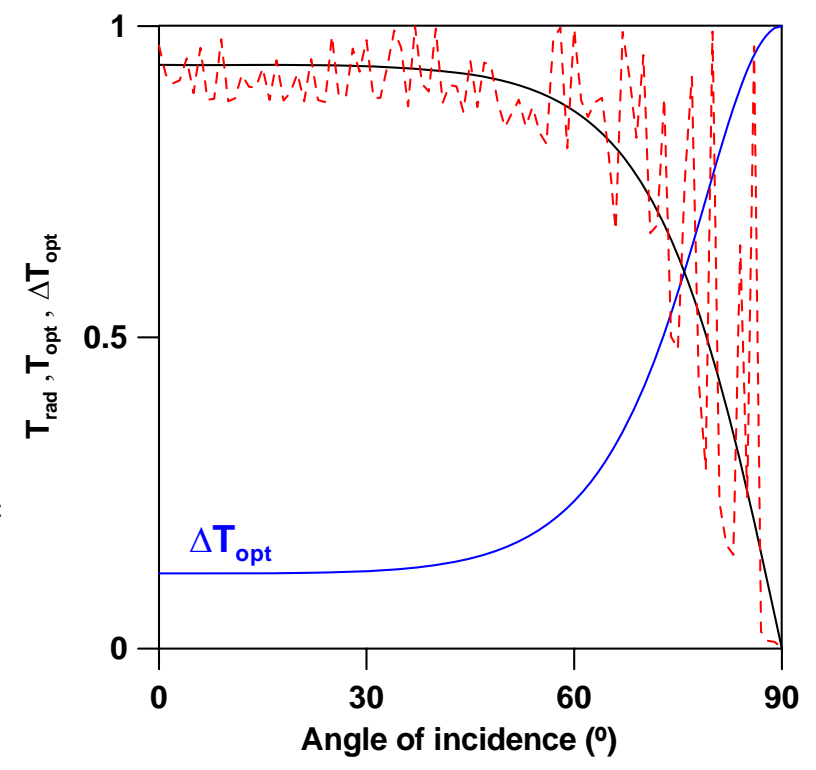

Figure 5. $T_{\text {rad }}, T_{\text {opt }}(d=0.002 \mathrm{~m})$ and maximum dispersion of $T_{o p t}$ as a function of angle of incidence for an uncoated calcium fluoride window at $486 \mathrm{~nm}$. The dashed lines are the optical values and the black straight lines are $\mathrm{T}_{\mathrm{rad}}$.

At first sight, and due to the fact that the optical equations (21) have been obtained taking into account interference processes, it may seem that performing some kind of averaging to the phase difference $\delta$ $\left(\delta=\frac{4 \pi}{\lambda}\right.$ nd $\left.\cos \theta\right)$ in the optical equations can lead us to the equations (18). But, what type of average? By supposing a homogeneous and isotropic material, the net variation of $\delta$ can be expressed as the following expression:

$$
\Delta \delta=4 \pi\left(\mathrm{n} \frac{\Delta \mathrm{d}}{\lambda}|\cos \theta|+\mathrm{n} \frac{\Delta \lambda}{\lambda^{2}} \mathrm{~d}|\cos \theta|+\mathrm{n} \frac{\mathrm{d}}{\lambda}|\sin \theta| \Delta \theta\right)
$$
value, if:

Hence, we conclude that the phase differences produced by the waves inside the plate can adopt any 
i) The plate surfaces are rough, meaning that the thickness $(\Delta d)$ and the surface normal $(\Delta \theta)$ vary randomly

ii) The incident light is a non-monochromatic radiation with a spectral bandwidth $(\Delta \lambda)$.

By assuming that any of these effects take place, the $R_{\text {opt }}$ and $T_{\text {opt }}$ could be averaged over all possible values of $\delta$. If the following averages are performed:

$$
\begin{aligned}
& <\mathrm{R}>=\frac{1}{2 \pi} \int_{0}^{2 \pi} \mathrm{R}_{\mathrm{opt}} \mathrm{d} \delta=\frac{1}{2 \pi} \int_{0}^{2 \pi} \frac{2(1-\cos \delta) \rho}{1+\rho^{2}-2 \rho \cos \delta} \mathrm{d} \delta \\
& <\mathrm{T}\rangle=\frac{1}{2 \pi} \int_{0}^{2 \pi} \mathrm{T}_{\mathrm{opt}} \mathrm{d} \delta=\frac{1}{2 \pi} \int_{0}^{2 \pi} \frac{(1-\rho)^{2}}{1+\rho^{2}-2 \rho \cos \delta} \mathrm{d} \delta
\end{aligned}
$$

we obtain $\left\langle R>=R_{\text {rad }}\right.$ and $\langle T\rangle=T_{\text {rad }}$, that is, the optical equations averaged over $\delta$ become into the radiation expressions for reflectance and transmittance. So, clearly distinguishing these two types of magnitudes is very important not only from a basic physical point of view but from a practical viewpoint. Accurate reflectance and transmittance measurements are necessary for calibration spectrometers or for determination of the optical properties of materials. It is also important to distinguish both magnitudes in optics catalogues ${ }^{19}$, where the transmittances of the colored glass filters, the neutral density filters, the interference filters, the uncoated windows, etc., are shown. Evidently, the expressions for transmittance which describe the corresponding behaviours are different for each type of filter; therefore we must take into account the above considerations, such as, the roughness of the plate or the spectral bandwidth of the radiation in order to correctly interpret the given information.

\section{CONCLUSIONS}

1) The definition given in Electromagnetic and Optics textbooks of the "intensity" as the time average of the amount of energy which crosses per second a unit area perpendicular to the direction of the flow is the radiometric magnitude irradiance only if the direction of the propagation is perpendicular to the surface.

2) The reflectance $\rho$ at an interface can always be expressed as the ratio of the reflected radiometric magnitude to the incident one. In contrast, the transmittance $\tau$ expression changes with the radiometric magnitude. Furthermore, the decreasing exponential law of the radiation propagating within an absorbing medium depends on the radiometric magnitude used. The more conventional version of this law corresponds to the case of a collimated beam of radiation, expressed as a function of irradiance. In this situation, the simple exponential law is satisfied with every radiometric magnitude. For other cases, the expression of the law may change. So one has to be very careful while interpreting this very well known equation.

3) Equations for reflectance $R$ and transmittance $T$ have been obtained for the case of a plane parallel plate by computing the total power flux (reflected and transmitted) at each interface. These expressions show a dependence on radiometric magnitudes similar to the one displayed by the reflectance $\rho$ and the transmittance $\tau$ at an interface. Only if the plate is surrounded by the same medium, $R$ and $T$ can be defined as the ratio of the reflected (transmitted) to the incident radiometric magnitude, no matter which one is chosen. The expressions obtained for this simple case have been compared with the corresponding ones found in Optics textbooks. Not only the mathematical expressions differ, but also the inner physical meaning, since the effect of interferences is only considered in the optical view. Both expressions (the radiation ones and the optical ones) coincide if the plate does not have smooth and parallel surfaces and/or the radiation presents a broad spectral bandwidth. 


\section{ACKNOWLEDGMENTS}

This work was supported by the institutions Ministerio de Educación y Ciencia, Ministerio de Ciencia e Innovación, Universidad del País Vasco/Euskal Herriko Unibertsitatea, Gobierno Vasco/Eusko Jaurlaritza, Diputación Foral de Bizkaia/Bizkaiko Foru Aldundia, and the European Union 7th Research Framework Programme, under projects TEC2006-13273-C03-01, PSS-370000-2008-39, UE08/16, S-PE08CA01, DIPE08/24, and CE07/12-AISHA II, respectively.

\section{REFERENCES}

[1] Cheng D K1983 Field and Wave Electromagnetics (Massachusetts: Addison-Wesley Publishing Company) [2] Heald M A and Marion J B 1995 Classical Electromagnetic Radiation (Saunders Collegue Publihshing)

[3] Jackson J D 1980 Electrodinámica clásica ( Madrid: Alhambra Universidad)

[4] Lorrain P and Corson DR 1979 Campos y ondas Electromagnéticos (Madrid : Selecciones científicas)

[5] Reitz J R and Milford F J 1972 Fundamentos de la teoría electromagnética (Mexico: Unión tipográfica editorial hispano-americana)

[6] Ulaby F T 1999 Applied Electromagnetics (New Yersey:Prentice Hall)

[7] Wangsness R K 1987 Campos Electromagnéticos.( Mexico: Ed. Limusa)

[8] Annequin R and Boutigny J 1976 Optica (Barcelona: Ed. Reverte)

[9] Born M and Wolf E 1980 Principles of Optics (Oxford: Pergamon Press)

[10] Casas J 1985 Optica (Zaragoza: cooperativa de artes gráficas)

[11] Ditchburn R W 1982 Optica (Barcelona: Ed. Reverte)

[12] Guenther R 1990 Modern Optics (John Wiley\&Sons)

[13] Heavens O S and Ditchburn R W 1991 Insigth to Optics ( Ed. Wiley)

[14] Hecht E and Zajac A 1986 Optica (Wilmington USA: Ed: Addison-Wesley Iberoamericana)

[15] Jenkins F A and White H E 1981 Fundamentals of Optics (Ed McGraw-Hill)

[16] Landsberg G S 1984 Optica (Moscu: Ed Mir)

[17] Pedrotti F L and Pedrotti L S 1993 Introduction to Optics (New Yersey:Prentice Hall)

[18] Nicodemus FE 1963 Radiance Am. J. Phys. 31 368-377

[19] Newport Resource Catalog (2008) 